TRANSACTIONS OF THE

AMERICAN MATHEMATICAL SOCIETY

Volume 363, Number 2, February 2011, Pages 1007-1022

S 0002-9947(2010)05175-X

Article electronically published on September 17, 2010

\title{
DISTINGUISHING BING-WHITEHEAD CANTOR SETS
}

\author{
DENNIS GARITY, DUŠAN REPOVŠ, DAVID WRIGHT, AND MATJAŽ ŽELJKO
}

\begin{abstract}
Bing-Whitehead Cantor sets were introduced by DeGryse and Osborne in dimension three and greater to produce examples of Cantor sets that were nonstandard (wild), but still had a simply connected complement. In contrast to an earlier example of Kirkor, the construction techniques could be generalized to dimensions greater than three. These Cantor sets in $S^{3}$ are constructed by using Bing or Whitehead links as stages in defining sequences. Ancel and Starbird, and separately Wright, characterized the number of Bing links needed in such constructions so as to produce Cantor sets. However it was unknown whether varying the number of Bing and Whitehead links in the construction would produce nonequivalent Cantor sets. Using a generalization of the geometric index, and a careful analysis of three dimensional intersection patterns, we prove that Bing-Whitehead Cantor sets are equivalently embedded in $S^{3}$ if and only if their defining sequences differ by some finite number of Whitehead constructions. As a consequence, there are uncountably many nonequivalent such Cantor sets in $S^{3}$ constructed with genus one tori and with a simply connected complement.
\end{abstract}

\section{BACKGROUND}

Two Cantor sets $X$ and $Y$ in $S^{3}$ are equivalent if there is a self homeomorphism of $S^{3}$ taking $X$ to $Y$. If there is no such homeomorphism, the Cantor sets are said to be inequivalent, or inequivalently embedded.

There has been an extensive study in the literature of nonstandard Cantor sets in $S^{3}$ (those that are not equivalent to the standard middle thirds Cantor set). Recent interest is partly due to the fact that such Cantor sets are often the invariant sets of certain dynamical systems. See [BC87, GRŽ05].

Antoine Ant20 constructed the first example of a nonstandardly embedded Cantor set. Sher [She68] showed that there were uncountably many inequivalent Cantor sets in $S^{3}$ by varying the number of components in the Antoine construction. These Cantor sets all had a nonsimply-connected complement and so were nonstandard.

Kirkor Kir58 constructed the first nonstandard Cantor set in $R^{3}$ with a simply connected complement. Any Cantor set in $R^{3}$ with a simply connected complement has the property that any 2 points in the Cantor set can be separated by a 2 -sphere missing the Cantor set (see [Sko86]). This allows the components of the stages of a defining sequence to be separated and makes the nonequivalence to the standard Cantor set much more difficult to detect. DeGryse and Osborne DO74] used a

Received by the editors October 19, 2008 and, in revised form, July 1, 2009.

2000 Mathematics Subject Classification. Primary 54E45, 54F65; Secondary 57M30, 57N10.

Key words and phrases. Cantor set, wild Cantor set, Bing link, Whitehead link, defining sequence. 
generalization of the Bing-Whitehead construction to produce nonstandard Cantor sets with a simply connected complement in all dimensions greater than or equal to three.

Ancel and Starbird AS89 and Wright Wri89 analyzed exactly which BingWhitehead constructions yielded Cantor sets. It was unknown whether changing the number of Bing and Whitehead links in the construction would yield inequivalent Cantor sets. In his dissertation Željko [Žel00] conjectured that if two BingWhitehead constructions yielded equivalent Cantor sets, then the constructions differed in a finite number of Whitehead constructions. This is essentially Question 7 in GR07. In this paper, we prove that this conjecture is true.

See [Shi74]), Bla51, Žel05, ZZel01, GRŽ05, and the bibliography in GR07 for additional examples of nonstandard Cantor sets. Robert Myers Mye88 has a very interesting paper on contractible 3 -manifolds that uses techniques very similar to the ones used in this paper, even though there are no Cantor sets mentioned.

In the next section we list the terminology and notation that we use and list the properties of Bing and Whitehead links from Wright's paper Wri89] that are needed in our analysis. We also list the main result that we obtain. In Section 3 we list the results on geometric linking and geometric index that we need. The results in this section follow from a generalization of Schubert's Sch53 results to links with more that one component. In Section 4 we prove that the boundaries of the stages in the construction for a Bing-Whitehead compactum can be made disjoint from boundaries of another defining sequence for the same compactum. In Section 5 we prove the main result. We end with some additional questions.

\section{Properties of Bing and Whitehead Links}

2.1. Bing and Whitehead links. We work in the piecewise linear category. A link is the finite union of disjoint simple closed curves. A torus is a 2-manifold homeomorphic to the product of two simple closed curves. A solid torus is a 3manifold homeomorphic to a disk cross a simple closed curve. We denote the interior and boundary of a manifold $M$ by $\operatorname{Int} M$ and $\partial M$, respectively. Let $T$ be a solid torus. Throughout this paper, we assume that the tori we are working with are unknotted in $S^{3}$. (The results and constructions also work in $R^{3}$.) A Bing link in $T$ is a union of 2 linked tori $F_{1} \cup F_{2}$ embedded in $T$ as shown in Figure1, A Whitehead link in $T$ is a torus $W$ embedded in $T$ as shown in the figure. For background details and terminology, see Wright's paper [Wri89. The link terminolgy arises from the link consisting of the cores of the interior tori together with a meridional curve on the outer torus.

2.2. Construction of Bing-Whitehead compacta. For completeness and consistency of notation, we outline the steps in the construction of Bing-Whitehead compacta. Let $M_{0}$ be an unknotted torus in $S^{3}$, and let $M_{1}$ be obtained from $M_{0}$ by placing a Bing construction in $M_{0}$. Inductively obtain $M_{k}$ from $M_{k-1}$ by placing a Bing construction in each component of $M_{k-1}$ or by placing a Whitehead construction in each component of $M_{k-1}$. Let $n_{1}$ be the number of consecutive Bing stages in the construction before the first Whitehead stage, and let $n_{k}$ be the number of consecutive Bing stages placed between the $(k-1)$ st and $k$ th Whitehead stages of $M$. 

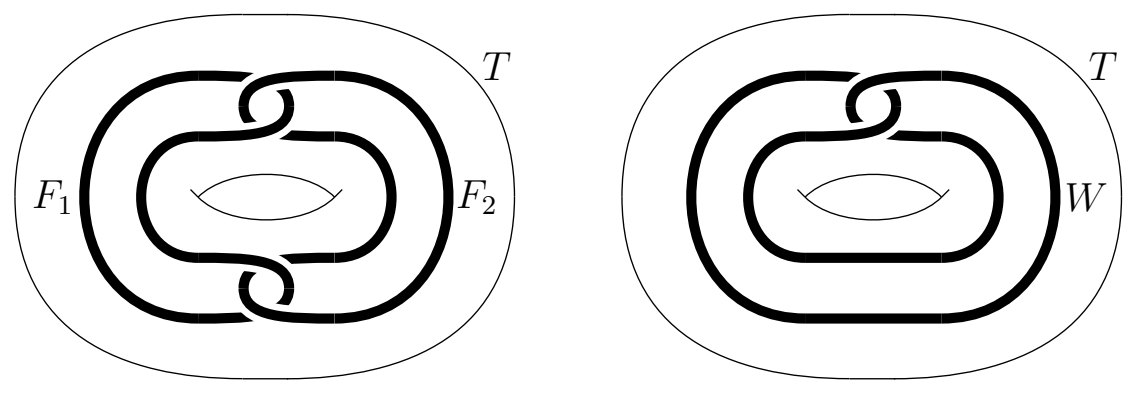

FiguRE 1. Bing and Whitehead constructions

Definition 2.1. The Bing-Whitehead compactum associated with this construction is defined to be

$$
X=\bigcap_{i=1}^{\infty} M_{i} \text { and is denoted } X=B W\left(n_{1}, n_{2}, \ldots\right) .
$$

We also define $M_{i}, i<0$, so that $M_{i}$ is a Whitehead construction in $M_{i-1}$ and let $X^{\infty}$ be $\bigcap_{i}\left(S^{3} \backslash M_{i}\right) . X^{\infty}$ is called the compactum at infinity associated with $X$. We assume that infinitely many of the $M_{i}, i>0$, arise from Bing constructions and that infinitely many of them arise from Whitehead constructions.

It is known AS89, Wri89 that this construction can be done so as to yield a Cantor set if and only if the series $\sum_{i} n_{i} 2^{-i}$ diverges. Specifically, if $G$ is the decomposition of $S^{3}$ consisting of the components of $X=B W\left(n_{1}, n_{2}, \ldots\right)$ and the remaining points of $S^{3}$, then $S^{3} / G$ is homeomorphic to $S^{3}$ if and only if this condition holds. The image of $X$ under the quotient map is then a Cantor set in $S^{3}$ called a Bing-Whitehead Cantor set. Standard results from decomposition theory Dav86 then imply that in this case, the construction of $X$ can be done so that the components of $X$ are points and thus $X$ itself is a Cantor set.

We introduce one additional definition that will be needed in the proof of the main theorem in Section 5

Definition 2.2. Suppose $X$ is a BW compactum with defining sequence $\left(M_{i}\right), i \geq$ 0 . The $\mathbf{B W}$ pattern for $X$ with respect to $\left(M_{i}\right)$ is the sequence $\left(\alpha_{1}, \alpha_{2}, \alpha_{3}, \ldots\right)$, where $\alpha_{i}=1$ if $M_{i}$ is obtained from $M_{i-1}$ by placing a Whitehead construction in each component, and where $\alpha_{i}=2$ if $M_{i}$ is obtained from $M_{i-1}$ by placing a Bing construction in each component.

2.3. Geometric properties. We list the key results from Wright's paper that will be needed in what follows.

Lemma 2.3. Let $M$ be a Bing or Whitehead link in a solid torus $T$.

- Wri89, Lemma 4.1] $T-\operatorname{Int} M$ is boundary incompressible; i.e., there is no 2 -disk $D \subset T-\operatorname{Int} M$ such that $D \cap(\partial T \cup \partial M)=\partial D$ with $\partial D$ essential in $\partial T \cup \partial M$.

- Wri89, Lemma 4.2] There is no annulus inside T connecting essential loops on two different components of $\partial M \cup \partial T$. 
Lemma 2.4. Let $X$ be a Bing-Whitehead compactum and $X^{\infty}$ the associated continuum at infinity.

- Wri89, Theorem 4.6] No sphere in the complement of $X \cup X^{\infty}$ separates $X \cup X^{\infty}$.

- Wri89, Theorem 4.3] A loop on the boundary of $M_{i}$ is essential in the boundary of $M_{i}$ if and only if it is essential in the complement of $X \cup X^{\infty}$.

- Wri89, Theorem 4.4] If loops $\ell_{1}$ and $\ell_{2}$ in $\partial M_{i}$ and $\partial M_{j}$, respectively, $i \neq$ $j$, are homotopic in the complement of $X \cup X^{\infty}$, then they are inessential in $X \cup X^{\infty}$.

2.4. Main result. Our ultimate goal is to determine when two Bing-Whitehead constructions $\left(M_{i}\right)$ and $\left(N_{j}\right)$ yield Cantor sets $X_{1}$ and $X_{2}$ that are equivalently embedded.

Theorem 2.5 (Main Theorem). Let $X_{1}$ be a Bing-Whitehead Cantor set associated with a defining sequence $\left(M_{i}\right)$ and let $X_{2}$ be a Bing-Whitehead Cantor set associated with a defining sequence $\left(N_{j}\right)$. If $X_{1}$ and $X_{2}$ are equivalently embedded, then the defining sequences differ in a finite number of Whitehead constructions. Specifically, if $X_{1}=B W\left(m_{1}, m_{2}, \ldots\right)$ with respect to $M_{i}$ and $X_{2}=B W\left(n_{1}, n_{2}, \ldots\right)$ with respect to $N_{j}$, then there are integers $p$ and $q$ such that $\sum_{i=1}^{p} m_{i}=\sum_{j=1}^{q} n_{j}$ and $m_{p+k}=n_{q+k}$ for all $k \geq 1$.

Remark 2.6. Note that the converse of Theorem 2.5 is also true. This was also observed in Željko's dissertation Žel00. Assume there are integers $p$ and $q$ such that $\sum_{i=1}^{p} m_{i}=\sum_{j=1}^{q} n_{j}$ and $m_{p+k}=n_{q+k}$ for all $k \geq 1$. Then there are homeomorphisms of $h_{1}$ and $h_{2}$ of $S^{3}$ taking $M_{p}$ and $N_{q}$ onto a collection of $2^{\sum_{i=1}^{p} m_{i}}$ pairwise disjoint, unknotted and unlinked tori. Using the fact that $m_{p+k}=n_{q+k}$ for all $k$, one can construct inductively homeomorphisms that take the components of $\left(N_{q+k}\right)$ onto the components of $h_{1}\left(M_{p+k}\right)$. Because $X_{1}$ and $X_{2}$ are Cantor sets, these homeomorphisms can be chosen so that the limit is a homeomorphism of $S^{3}$ to itself taking $X_{2}$ to $h_{1}\left(X_{1}\right)$

Corollary 2.7. There are uncountably many inequivalent Bing-Whitehead Cantor sets in $S^{3}$.

Proof. To get uncountably many distinct examples, start with the example

$$
B W\left(1,2,4, \ldots, 2^{i}, 2^{i+1}, \ldots\right) \text {. }
$$

Let $\alpha=\left(j_{0}, j_{1}, j_{2}, \ldots\right)$ be an increasing sequence of positive integers. The examples we seek are of the form

$$
B W\left(1+3^{j_{0}}, 2+3^{j_{1}}, 4+3^{j_{2}}, \ldots, 2^{i}+3^{j_{i}}, 2^{i+1}+3^{j_{i+1}}, \ldots\right) .
$$

By Theorem 2.5. for distinct sequences of increasing integers, no two of these are equivalent.

\section{Algebraic And geometric index}

3.1. Algebraic index. If $S$ is a solid torus in another solid torus $T$, the algebraic index of $S$ in $T$ is $|\alpha|$, where $\alpha$ is the integer in $H_{1}(T)$ represented by the center line of $S$. The algebraic index is multiplicative, so that if $S \subset T \subset U$ are solid tori, the algebraic index of $S$ in $U$ is the product of the algebraic index of $S$ in $T$ with the algebraic index of $T$ in $U$. Note that the algebraic index of a Whitehead link in 
the torus containing it is 0 , as is the algebraic index of each component of a Bing link.

3.2. Geometric index. If $K$ is a link in the interior of a solid torus $T$, then we denote the geometric index of $K$ in $T$ by $\mathrm{N}(K, T)$. The geometric index is the minimum of $|K \cap D|$ over all meridional disks $D$ of $T$. A core of a solid torus $T$ in 3-space is a simple closed curve $J$ so that $T$ is a regular neighborhood of $J$. Likewise, a core for a finite union of disjoint solid tori is a link consisting of one core from each of the solid tori. If $T$ is a solid torus and $M$ is a finite union of disjoint solid tori so that $M \subset$ Int $T$, then the geometric index $\mathrm{N}(M, T)$ of $M$ in $T$ is $\mathrm{N}(K, T)$ where $K$ is a core of $M$. The geometric index of a Bing link $F_{1} \cup F_{2}$ in a torus $T$ is 2 . The geometric index of a Whitehead link $W$ in a torus $T$ is also 2 .

Theorem 3.1. Let $T_{0}$ and $T_{1}$ be unknotted solid tori in $S^{3}$ with $T_{0} \subset \operatorname{Int} T_{1}$ and $N\left(T_{0}, T_{1}\right)=1$. Then $\partial T_{0}$ and $\partial T_{1}$ are parallel; i.e., the manifold $T_{1}-\operatorname{Int} T_{0}$ is homeomorphic to $\partial T_{0} \times I$, where $I$ is the closed unit interval $[0,1]$.

Proof. The proof follows from work of Schubert Sch53] and regular neighborhood theory. Let $J$ be a core of $T_{0}$. Since $T_{0}$ is unknotted, $J$ is an unknotted simple closed curve. The geometric index of $J$ in $T_{1}$ is one. By Schubert, $J$ is either a core of $T_{1}$ or a sum of knots with a core. Since $J$ is unknotted, $J$ must be a core of $T_{1}$. Since $J$ is a core of both $T_{0}$ and $T_{1}$, regular neighborhood theory [RS72] shows that $T_{1}-\operatorname{Int} T_{0}$ is homeomorphic to $\partial T_{0} \times I$.

Theorem 3.2. Let $T_{0}$ be a finite union of disjoint solid tori. Let $T_{1}$ and $T_{2}$ be solid tori so that $T_{0} \subset \operatorname{Int} T_{1}$ and $T_{1} \subset \operatorname{Int} T_{2}$. Then $N\left(T_{0}, T_{2}\right)=N\left(T_{0}, T_{1}\right) \cdot N\left(T_{1}, T_{2}\right)$.

Proof. Schubert proves the case where $T_{0}$ is a single solid torus, but his proof works for the above case with no changes.

There is one additional result we will need in Section 4 .

Theorem 3.3. Let $T$ be a solid torus in $S^{3}$ and let $T_{1}, T_{2}$ be unknotted solid tori in $T$, each of geometric index 0 in $T$. Then the geometric index of $\bigcup_{i=1}^{2} T_{i}$ in $T$ is even.

Proof. If the geometric index were odd, then there is a meridional disk $D$ of $T$ that intersects the cores of $T_{1} \cup T_{2}$ transversally an odd number of times. So this means that $D$ must intersect the core of either $T_{1}$ or $T_{2}$ an odd number of times. But if a meridional disk of $T$ intersects a simple closed curve $J$ transversally an odd number of times, the algebraic index of $J$ in $T$ is odd and so $J$ is essential in $T$. However, the cores of the $T_{i}$ are both inessential because they lie in a ball in $T$.

3.3. Boundary parallel tori. The next three results make use of the material on geometric index to determine when the boundaries of certain tori are parallel. These results are used in the proof of the main theorem in Section 5 to inductively match up stages in different Bing-Whitehead defining sequences.

Theorem 3.4. Let $W$ be a Whitehead link in the solid torus $T$ in $S^{3}$. If $T^{\prime} \subset T$ is a solid unknotted torus whose boundary separates $\partial W$ from $\partial T$, then $\partial T^{\prime}$ is parallel to either $\partial W$ or $\partial T$.

Proof. Since $\partial T^{\prime}$ separates $\partial W$ from $\partial T$, we have $W \subset \operatorname{Int} T^{\prime}$ and $T^{\prime} \subset \operatorname{Int} T$. Since $\mathrm{N}\left(W, T^{\prime}\right) \cdot \mathrm{N}\left(T^{\prime}, T\right)=\mathrm{N}(W, T)=2$, either $\mathrm{N}\left(W, T^{\prime}\right)=1$ or $\mathrm{N}\left(T^{\prime}, T\right)=1$. The conclusion now follows from Theorem 3.2 . 
Theorem 3.5. Let $F_{1} \cup F_{2}$ be a Bing link in a solid torus $T$ in $S^{3}$. If $T^{\prime} \subset T$ is a solid unknotted torus whose boundary separates $\partial\left(F_{1} \cup F_{2}\right)$ from $\partial T$, then $\partial T^{\prime}$ is parallel to $\partial T$.

Proof. Since $\partial T^{\prime}$ separates $\partial\left(F_{1} \cup F_{2}\right)$ from $\partial T$, we have $F_{1} \cup F_{2} \subset \operatorname{Int} T^{\prime}$ and $T^{\prime} \subset$ Int $T$. Since $\mathrm{N}\left(F_{1} \cup F_{2}, T^{\prime}\right) \cdot \mathrm{N}\left(T^{\prime}, T\right)=\mathrm{N}\left(F_{1} \cup F_{2}, T\right)=2$, either $\mathrm{N}\left(F_{1} \cup F_{2}, T^{\prime}\right)=1$ or $\mathrm{N}\left(T^{\prime}, T\right)=1$. We show that $\mathrm{N}\left(F_{1} \cup F_{2}, T^{\prime}\right)=1$ is impossible. Suppose $\mathrm{N}\left(F_{1} \cup F_{2}, T^{\prime}\right)=1$; then $\mathrm{N}\left(F_{i}, T^{\prime}\right)=1$ for either $i=1$ or $i=2$. Now $0=\mathrm{N}\left(F_{i}, T\right)=\mathrm{N}\left(F_{i}, T^{\prime}\right) \cdot \mathrm{N}\left(T^{\prime}, T\right)=\mathrm{N}\left(T^{\prime}, T\right) \neq 0$, a contradiction. So we conclude that $\mathrm{N}\left(T^{\prime}, T\right)=1$, and the conclusion now follows from Theorem 3.2 .

Theorem 3.6. Let $F_{1} \cup F_{2}$ be a Bing link in the solid torus $T$ in $S^{3}$. If $S$ is the boundary of a solid unknotted torus that separates $\partial F_{1} \cup \partial F_{2} \cup \partial T$, then $S$ is parallel to one of $\partial F_{1}, \partial F_{2}, \partial T$.

Proof. If $S$ separates $\partial T$ from $\partial F_{1} \cup \partial F_{2}$, then we can invoke the previous theorem. The other cases follow from the fact that there are homeomorphisms of $S^{3}$ to itself that take $T-\operatorname{Int}\left(F_{1} \cup F_{2}\right)$ to itself and take $\partial F_{i}$ to $\partial T$. These homeomorphisms follow from the (well-known) fact that $F_{1} \cup F_{2} \cup\left(S^{3}-\operatorname{Int} T\right)$ are Borromean rings.

\section{Boundary intersections of DEFINING SEQUENCES}

4.1. Setup. For the rest of this section, we assume that there is a Bing-Whitehead compactum $X$ with two defining sequences $\left(M_{k}\right)$ and $\left(N_{k}\right)$. Let $X_{M}^{\infty}$ be the continuum at infinity associated with the first defining sequence and let $X_{N}^{\infty}$ be the continuum at infinity associated with the second defining sequence.

Theorem 4.1. Let $X,\left(M_{k}\right),\left(N_{k}\right), X_{N}^{\infty}$, and $X_{M}^{\infty}$ be as above. Suppose that $i$ and $j$ are chosen so that

- $M_{i-1}$ is in $N_{1}$ and so is in the complement of $X_{N}^{\infty}$,

- $N_{j-1}$ is in $M_{1}$ and so is in the complement of $X_{M}^{\infty}$.

Let $n$ be a fixed integer. Then there is a homeomorphism $h$ of $S^{3}$ to itself, fixed on $X \cup\left(S^{3}-M_{1}\right) \cup\left(S^{3}-N_{1}\right)$, so that $h\left(\partial\left(M_{i+m}\right)\right) \cap \partial\left(N_{j+\ell}\right)=\emptyset$ for each nonnegative $m$ and $\ell$ less than $n$.

The remainder of this section is devoted to the proof of this theorem. We will need to apply the following lemmas. Note that Lemma 4.2 is the case $n=0$ of Theorem 4.1 .

Lemma 4.2. Let $X,\left(M_{k}\right),\left(N_{k}\right), X_{N}^{\infty}$, and $X_{M}^{\infty}$ be as above. Suppose that $i$ and $j$ are chosen so that

- $M_{i-1}$ is in $N_{1}$ and so is in the complement of $X_{N}^{\infty}$,

- $N_{j-1}$ is in $M_{1}$ and so is in the complement of $X_{M}^{\infty}$.

Then there is a homeomorphism $h$ of $S^{3}$ to itself, fixed on $X \cup\left(S^{3}-M_{1}\right) \cup\left(S^{3}-N_{1}\right)$, so that $h\left(\partial M_{i}\right) \cap \partial N_{j}=\emptyset$.

Lemma 4.3. Let $X,\left(M_{k}\right),\left(N_{k}\right), X_{N}^{\infty}$, and $X_{M}^{\infty}$ be as above. Suppose:

- $T^{\prime}$ is a component of $N_{j}$ and $N_{j}$ is in the complement of $X_{M}^{\infty}$,

- $M_{i} \cap T^{\prime} \subset \operatorname{Int}\left(T^{\prime}\right)$ and consists of components $T_{1}, \ldots, T_{r}$ of $M_{i}$.

Then there is a self homeomorphism $h$ of $S^{3}$, fixed on $X \cup\left(S^{3}-T^{\prime}\right)$, so that $h\left(\partial\left(\bigcup_{k=1}^{r} T_{k}\right)\right) \cap \partial\left(N_{j+1}\right)=\emptyset$. 
Lemma 4.4. Let $X,\left(M_{k}\right),\left(N_{k}\right), X_{N}^{\infty}$, and $X_{M}^{\infty}$ be as above. Suppose:

- $T$ is a component of $M_{i}$ and $M_{i}$ is in the complement of $X_{N}^{\infty}$,

- $N_{j} \cap T \subset \operatorname{Int}(T)$ and consists of components $T_{1}^{\prime}, \ldots, T_{r}^{\prime}$ of $N_{j}$.

Then there is a self homeomorphism $h$ of $S^{3}$, fixed on $X \cup\left(S^{3}-T\right)$, so that $h\left(\partial\left(M_{i+1}\right)\right) \cap \partial\left(\bigcup_{k=1}^{r} T_{k}^{\prime}\right)=\emptyset$.

4.2. Proof of Lemma 4.2. Adjust the components of $\partial M_{i}, \partial N_{j-1}, \partial N_{j}$, and $\partial N_{j+1}$ so that they are in general position. This implies that the boundaries of these components intersect in a finite collection of pairwise disjoint simple closed curves. We will successively remove these curves of intersection by homeomorphisms of $S^{3}$.

4.2.1. Removing trivial curves of intersection. Focus on one component $T$ of $M_{i}$. Consider $\partial T \cap \partial N_{j}$. This intersection, if nonempty, consists of a finite number of simple closed curves. By Lemma 2.4, and by the hypotheses of Lemma 4.2, one of these curves is inessential on $\partial T$ if and only if it is inessential on some component of $\partial N_{j}$. If there are any inessential curves, choose a component $T^{\prime}$ of $N_{j}$ that contains one in $\partial T^{\prime}$. Choose an innermost inessential simple closed curve $\alpha$ on $\partial T^{\prime}$. Since $\alpha$ is innermost, it bounds a disk $D^{\prime}$ with interior missing $\partial T$. The curve $\alpha$ also bounds a disk $D$ in $\partial T$.

The 2-sphere $D \cup D^{\prime}$ bounds a three-cell in $M_{1} \cap N_{1}$ that by Lemma 2.4 contains no points of $X$. Use this three-cell to push $D$ onto $D^{\prime}$ and then a little past $D^{\prime}$ into an exterior collar on the cell by a homeomorphism $h$ of $S^{3}$. This homeomorphism can be chosen to fix $X, S^{3}-M_{1}$, and $S^{3}-N_{1}$. This has the result that $h(\partial T) \cap \partial T^{\prime}$ has fewer curves of intersection than $\partial T \cap \partial T^{\prime}$, and so that no new curves of intersection with $\partial\left(N_{j}\right)$ are introduced. Continuing this process eventually removes all inessential curves of intersection on $\partial T$. Repeating this process for each component of $M_{i}$ removes all inessential curves of intersection of the boundaries of $M_{i}$ and $N_{j}$. Repeating the process with $N_{j-1}$ and $N_{j+1}$ completes the first step of the proof.

Therefore there is a homeomorphism $h_{1}$ of $S^{3}$ to itself, fixed on $X \cup X_{M}^{\infty} \cup X_{N}^{\infty}$ such that $h_{1}\left(\partial M_{i}\right) \cap\left(\partial N_{j-1} \cup \partial N_{j} \cup \partial N_{j+1}\right)$ has no nontrivial curves of intersection. To simplify notation in what remains, we will refer to $h_{1}\left(M_{i}\right)$ as (the new) $M_{i}$.

Remark 4.5. At this point, let $S$ be a component of $M_{i}$. Then there is at most one component $S^{\prime}$ of $N_{j}$ for which $\partial S \cap \partial S^{\prime} \neq \emptyset$, and if this is the case, then $\partial S \cap \partial N_{j-1}=\emptyset$ and $\partial S \cap \partial N_{j+1}=\emptyset$. This follows directly from Lemma 2.3. In fact, the curves of intersection on $\partial S$ must be parallel $(p, q)$ torus curves, and the corresponding curves on $\partial S^{\prime}$ must be parallel $(s, t)$ curves. If both $p$ and $q$ are greater than 1 , so that the torus curve is a nontrivial knot, then $(s, t)=(p, q)$ or $(s, t)=(q, p)$ by results from Rolfsen [Rol76, but we do not use this observation.

We now work towards removing these remaining curves of intersection of the boundaries, so that the components of $\left(M_{i}\right)$ under consideration either are contained in or contain the components of $\left(N_{j}\right)$ under consideration. Consider an annulus $A$ on the boundary of $S$ bounded by two adjacent curves of the intersection of $\partial S$ and $\partial S^{\prime}$. Choose this annulus so that its interior lies in the interior of $S^{\prime}$. We consider the separate possibilities for how the boundary curves of $A$ lie on $S^{\prime}$.

4.2.2. Curves of intersection on $S^{\prime}$ that are $(p, q)$ curves for $p \geq 2$. Consider a meridional disc $D$ for $S^{\prime}$ in general position with respect to $A$ so that $D \cap A$ 
consists of $p$ arcs intersecting the boundary of $D$ in endpoints and of simple closed curves. Figure 2 illustrates the situation when $p=5$ and $q=3$. The shaded regions indicate the intersection of the next stage $N_{j+1}$ with $D$.
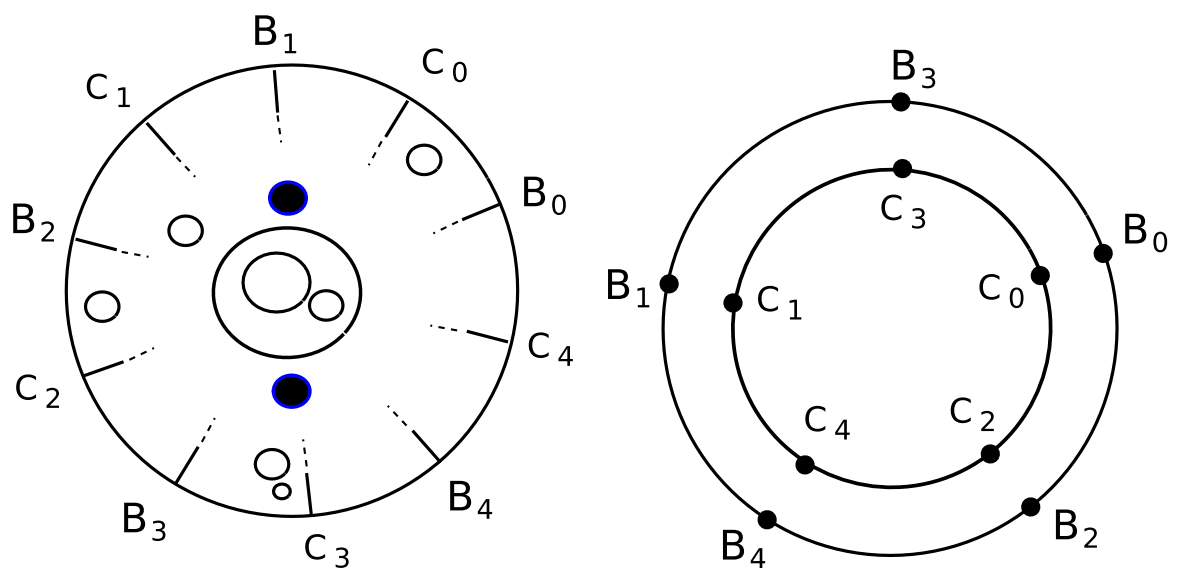

Figure 2. Meridional disc $D$ of $S^{\prime}$ and annulus $A$

Label the boundary curves of the annulus $A$ as curves $B$ and $C$. Label the intersection points of $B$ with the meridional disc $D$ sequentially around the boundary of $D$ as $B_{0}, B_{1}, \ldots, B_{p-1}$ and similarly label the intersection points of $C$ with $D$ as $C_{0}, C_{1}, \ldots, C_{p-1}$. Because $B$ and $C$ are parallel $(p, q)$ curves on the boundary of $S^{\prime}$, the intersection points $B_{i}$ and $C_{i}$ must alternate. We have not yet indicated how the arcs leaving the points $C_{i}$ and $B_{i}$ are connected.

The corresponding points on the annulus $A$ are labeled sequentially along the curve $B$ as $B_{0}, B_{q}, B_{2 q}, \ldots, B_{(p-1) q}$, where subscripts are taken $\bmod p$. The points on the annulus $A$ along the $C$ curve are similarly labeled sequentially $C_{0}, C_{q}, C_{2 q}$, $\ldots, C_{(p-1) q}$. Again, Figure 2 illustrates the case $p=5$ and $q=3$.

We will argue that the intersection of $A$ with $D$ can be adjusted using cut and paste techniques so that the end result is intersections as in one of the two cases in Figure 3 ,

Each of the regions labeled $M_{i}$ will be shown to be a meridional disc of a solid torus that is contained in $S^{\prime}$. This solid torus will then be used to push across and remove the intersections of $A$ with $D$.

Refer back to Figure 2. As a first step, in adjusting the intersection of $D$ and $A$ we show how to remove simple closed curves of intersection. Each simple closed curve is trivial in $A$; otherwise a $(p, q)$ curve for $p \geq 2$ on the boundary of $S^{\prime}$ would be null homotopic in $S^{\prime}$. None of the simple closed curves can enclose either or both of the shaded regions indicated because they are contractible in $A$ and thus contractible in $S^{\prime}$ missing $X$. Choosing an innermost such simple closed curve in $D$, the intersection can be removed by an argument similar to that used in removing trivial curves of intersection in the previous section. Specifically, there is a homeomorphism $h$ from $S^{3}$ to itself, fixed on $X$ and the complement of $S^{\prime}$ such that $h(A) \cap D$ has fewer simple closed curves of intersection than $A \cap D$ does. Inductively, all such simple closed curves of intersection can be removed by a self homeomorphism of $S^{3}$ fixed on $X$ and on $S^{3}-S^{\prime}$. 

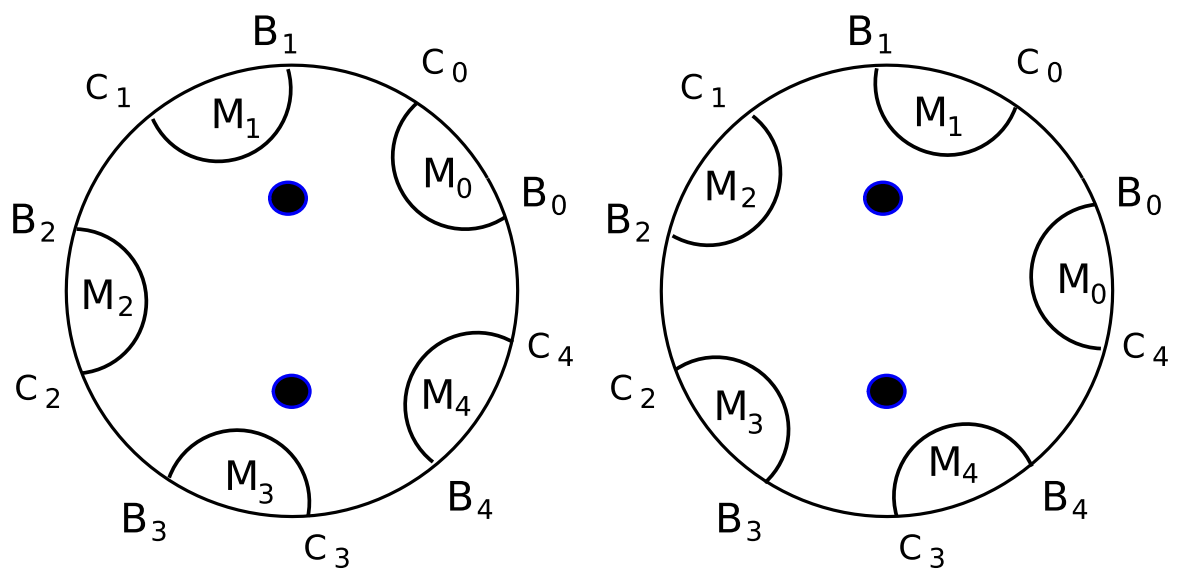

Figure 3. Meridional disc $D$ of $S^{\prime}$ after adjustment

After such simple closed curves of intersection are removed, we are left with the situation pictured in Figure 4. Again, we have not yet indicated how the arcs emanating form the boundary points are connected.

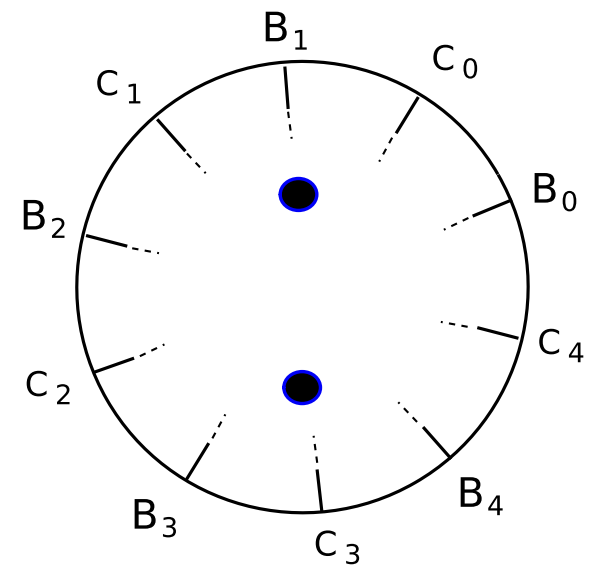

Figure 4. Disc $D$ of $S^{\prime}$ after simple closed curves removed

First note that if any $B_{i}$ were joined to a $B_{j}$, the arc joining them would separate the disc $D$ and leave an odd number of boundary points on both sides. Since the boundary points are joined in pairs, this is not possible. So each $B_{i}$ is joined to some $C_{j}$ by an arc of intersection of $A$ with $D$.

Next, consider these arcs in the annulus $A$ as in Figure 5 .

If point $B_{0}$ is joined by an arc of intersection to point $C_{k q}$, then each point $B_{i q}$ must be joined to the point $C_{(k+i) q}$. Otherwise it would not be possible to have disjoint arcs joining the points on $B$ to the points on $C$.

Now consider these arcs of intersection again in $D$ as in Figure 4 Since point $B$ with subscript $i q(\bmod p)$ is joined to point $C$ with subscript $(i+k) q(\bmod p)$, the 


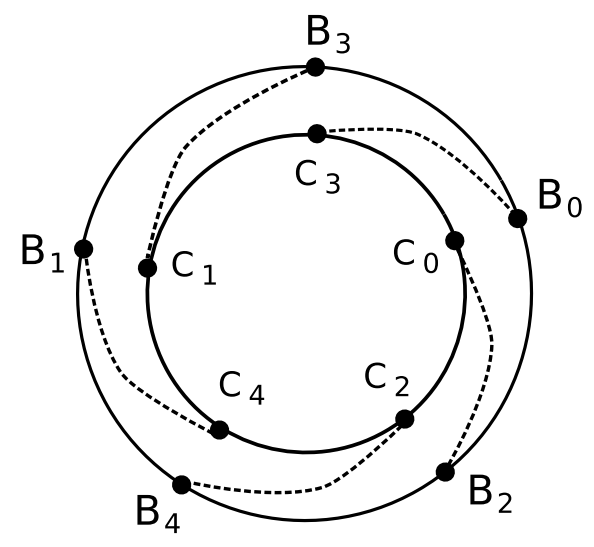

Figure 5. Annulus $A$ with $\operatorname{arcs}$ of intersection

difference in indices of any two of the joined points is $(i+k) q-i q(\bmod p)=k q$ $(\bmod p)$. Unless this difference is 0 or $p-1$, it is not possible to place the $p$ arcs in $D$ in a pairwise disjoint fashion. Thus either each $B_{i}$ in $D$ is joined by an arc to $C_{i}$ or each is joined by an arc to $C_{i-1(\bmod p)}$. Thus, the arcs of intersection are as pictured in Figure 3 ,

The intersection of the annulus $A$ with $\partial\left(S^{\prime}\right)$ separates $\partial\left(S^{\prime}\right)$ into two annuli. Let $A_{1}$ be the annulus whose intersection with $D$ consists of $p$ arcs joining the same points of the boundary of $D$ as the arcs of intersection of $A$ and $D$. Then $A \cup A_{1}=T_{1}$ is a torus. See Figure 6 for an illustration of this in one of the cases from Figure 3 .

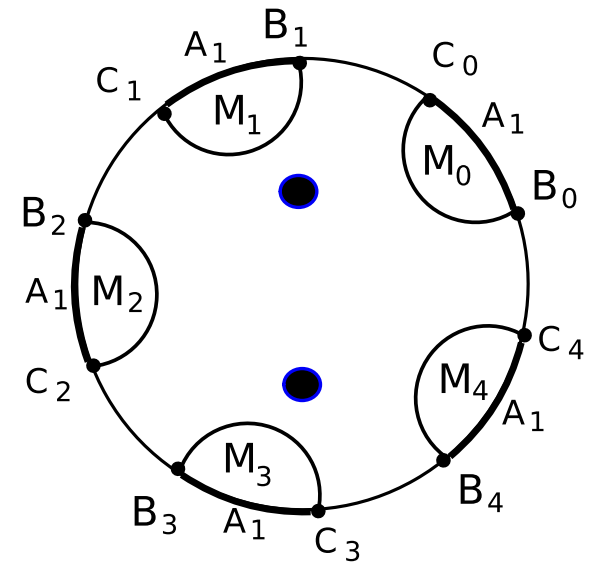

Figure $6 . D$ with regions $M$

Without loss of generality, $B_{0}$ is joined to $C_{0}$ by an arc $\alpha_{0}$ of the intersection of $A$ with $D$. Let $\beta_{0}$ be the arc in $A_{1}$ in the boundary of $D$ joining the endpoints of $\alpha_{0}$. The loop $\alpha_{0} \cup \beta_{0}$ is a nontrivial loop in $T_{1}$ and $T_{1}$ separates $S^{3}$ into two components. Let $D_{1}$ be the component that contains the disc $M_{0}$ in $D$ bounded 
by $\alpha_{0} \cup \beta_{0}$. Since $\alpha_{0} \cup \beta_{0}$ bounds a disc in $D_{1}, D_{1}$ is a solid torus by a standard argument. (See Rol76].)

We now show that the next stage of the construction in $S^{\prime}$ cannot intersect $D_{1}$. Notice that the geometric and algebraic index of $D_{1}$ in $S^{\prime}$ is $p \geq 2$. The geometric index of the next stage of $N$ in $S^{\prime}$ is 2 . If the next stage is a Whitehead construction $W$ in $S^{\prime}$ that lies in $D_{1}$, and the geometric index of $W$ in $D_{1}$ is 0 or $>1$, there is a contradiction by Theorem 3.2. If the geometric index of $W$ in $D_{1}$ is 1 , then the algebraic index of $W$ in $S^{\prime}$ is the same as the algebraic index of $D_{1}$ in $S^{\prime}$, which is $p \neq 0$, again a contradiction.

If the next stage of $S^{\prime}$ in $D$ is a Bing construction $B=F_{1} \cup F_{2}$ in $S^{\prime}$, and one component, say $F_{1}$, lies in $D_{1}$, then the geometric index of $F_{1}$ in $T_{1}$ must be zero because the geometric index of $F_{1}$ in $S^{\prime}$ is zero. If $F_{2}$ does not also lie in $D_{1}$, then $F_{1}$ lies in a ball that lies in $D_{1}$ and, hence, misses $F_{2}$, a contradiction. If both components of $B$ lie in $D_{1}$, then by Theorem 3.3 the geometric index of $B$ in $D_{1}$ is even and is thus 0 or $>1$. This implies by Theorem 3.2 that the geometric index of $B$ in $S^{\prime}$ is 0 or $\geq 4$, a contradiction.

The intersection of $S$ with $S^{\prime}$ corresponding to $A$ can now be removed by a homeomorphism of $S^{3}$ fixed on $X$ and on the complement of a small neighborhood of $S^{\prime}$ that takes $A$ through $D_{1}$ to an annulus parallel to $A_{1}$ and just outside of $S^{\prime}$. Inductively, all curves of intersection of $S$ with $S^{\prime}$ can be removed by a homeomorphism of $S^{3}$ fixed on $X$ and the complement of a small neighborhood of $S^{\prime}$.

4.2.3. Curves of intersection on $S^{\prime}$ that are $(p, q)$ curves for $p=1$. An argument similar to that in the preceding section can be used. After removing trivial curves of intersection, we are left with an intersection of $A$ with $S^{\prime}$ as pictured in Figure 7. $A$ divides $S^{\prime}$ into two tori, labeled $U$ and $V$ in the figure.

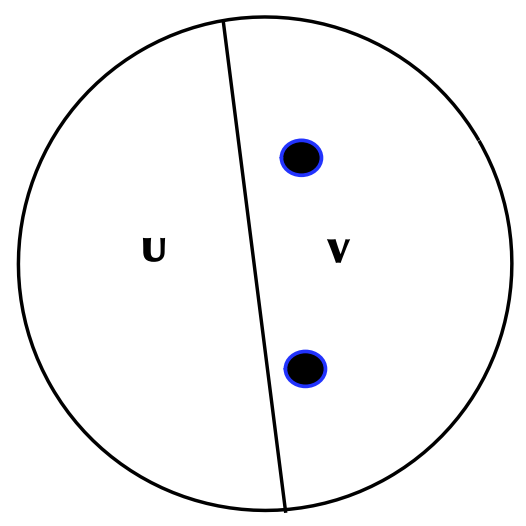

Figure 7 . The case $p=1$

The next stage of the construction is either in the solid torus labeled $U$ or in the solid torus labeled $V$. This is clear if the next stage is a Whitehead construction.

If the next stage of $S^{\prime}$ in $D$ is a Bing construction $B=F_{1} \cup F_{2}$ in $S^{\prime}$, and only one component, say $F_{1}$, lies in $V$, then the geometric index of $F_{1}$ in $V$ must be zero because the geometric index of $F_{1}$ in $S^{\prime}$ is zero. But then $F_{1}$ lies in a ball that lies in $V$ and, hence, misses $F_{2}$, a contradiction. So both components of the next stage 
lie entirely in $U$ or entirely in $V$. The intersection of $A$ with $D$ can be removed by pushing across the other torus.

4.2.4. Curves of intersection on $S^{\prime}$ that are $(p, q)$ curves for $p=0$. In this case the curve is a $(0, q)$ curve for the torus $S^{\prime}$, but it is a $(q, 0)$ curve for the complementary torus with $q \neq 0$. In this case there is an annulus $A$ on the boundary of $S$ that has its interior in the exterior of $S^{\prime}$, so that the intersection of $A$ with the boundary of $S^{\prime}$ consists of curves in the intersection of the boundaries of $S$ and $S^{\prime}$. We have essentially turned the problem inside out, and we can use the previous methods to push $A$ to the interior of $S^{\prime}$ fixed on a slightly shrunken $S^{\prime}$, all the other components of $M_{i}$, and the complement of $M_{i-1}$.

The discussion above completes the proof of Lemma 4.2 .

4.3. Proof of Lemmas 4.3 and 4.4. The proofs of these two lemmas are virtually identical, with $M$ and $N$ interchanged in the second lemma. For the proof of Lemma 4.3. under the assumption that $M_{i} \cap T^{\prime} \subset \operatorname{Int}\left(T^{\prime}\right)$ and consists of components $T_{1}, \ldots, T_{r}$ of $M_{i}$, one mimics the proof of Lemma 4.2, to make each boundary of $T_{i}$ disjoint from the boundaries of the one or two components of $N_{j+1}$ in $T^{\prime}$. The only additional step is taking care that each homeomorphism from the proof of Lemma 4.2 can be achieved by fixing $S^{3}-T^{\prime}$. This is clear because the 3-cells or tori used as guides for these homeomorphisms are all in the interior of $T^{\prime}$ and all miss $X$.

Remark 4.6. Note that the hypotheses of Lemma 4.3 give that the components of $M_{i}$ intersecting $T^{\prime}$ in $N_{j}$ are all interior to $T^{\prime}$, and so their boundaries miss the boundary of $T^{\prime}$ and thus the boundary of $N_{j}$. After the homeomorphism of the lemma, the boundaries of the components of $M_{i}$ under consideration miss the boundaries of both $N_{j}$ and $N_{j+1}$. The fact that the components are interior to $T^{\prime}$ implies that the boundaries of these components also miss all previous stages of $\left(N_{k}\right)$.

4.4. Proof of Theorem 4.1, By assumption, $i$ and $j$ are chosen so that $M_{i-1}$ is in $N_{1}$ and $N_{j-1}$ is in $M_{1}$. Let $n$ be a fixed integer. By Lemma 4.2, there is a homeomorphism $h_{1}$ of $S^{3}$ to itself, fixed on $X \cup\left(S^{3}-M_{1}\right) \cup\left(S^{3}-N_{1}\right)$, so that $h_{1}\left(\partial\left(M_{i}\right)\right) \cap \partial\left(N_{j}\right)=\emptyset$. This implies that each component $S$ of $h_{1}\left(M_{i}\right)$ is either contained in the interior of a component of $S^{\prime}$ of $N_{j}$, or contains components of $N_{j}$.

Assume that $S$ is contained in a component $S^{\prime}$ of $N_{j}$. By Lemma 4.3. there is a homeomorphism $h_{2}$ of $S^{3}$, fixed on $X$ and the complement of $S^{\prime}$ so that $\partial\left(h_{2}(S)\right)$ does not intersect $\partial\left(N_{j}\right) \cup \partial\left(N_{j+1}\right)$. Either $h_{2}(S)$ is contained in a component $S^{\prime \prime}$ of $N_{j+1}$ or it contains components of $N_{j+1}$.

Continue inductively applying Lemma 4.3 until a stage is reached so that the image of $S$ under the composition of the homeomorphisms at each stage, $h(S)$, contains components $T_{1}^{\prime}, \ldots, T_{r}^{\prime}$ of some $N_{j+\ell}$, and so that $\partial(h(S))$ does not intersect $\partial\left(N_{j}\right) \cup \partial\left(N_{j+1}\right) \cup \ldots \cup \partial\left(N_{j+\ell}\right)$. Such a stage must be reached because every time a Bing construction occurs in the defining sequence $\left(N_{k}\right)$, components of $\left(N_{k}\right)$ at that stage contain fewer components of the image of $M_{i}$ than at the previous stage.

At this point, apply Lemma 4.4 to get a homeomorphism $h^{\prime}$ of $S^{3}$, fixed on $X$ and on the complement of $h(S)$, so that $h^{\prime} \circ h\left(\partial M_{i+1}\right) \cap \partial\left(\bigcup_{k=1}^{r} T_{k}^{\prime}\right)=\emptyset$. We then have that the boundaries of $h^{\prime} \circ h(S)$ and the boundaries of $h^{\prime} \circ h$ of all components of $M_{i+1}$ contained in $X$ are disjoint from $\partial\left(N_{j}\right) \cup \partial\left(N_{j+1}\right) \cup \ldots \cup \partial\left(N_{j+\ell}\right)$.

Do the above procedure for each component of $h_{1}\left(M_{i}\right)$ that is contained in a component of $N_{j}$. Do a similar procedure, starting with Lemma 4.4 for each 
component of $h_{1}\left(M_{i}\right)$ containing components of $N_{j}$. The result is a homeomorphism $h_{3}$ of $S^{3}$, fixed on $X$ and on the complement of $h_{1}\left(M_{i}\right) \cup N_{j}$, so that

$$
h_{3} \circ h\left(\partial M_{i} \cup \partial M_{i+1}\right) \cap\left(\partial N_{j} \cup \partial N_{j+1}\right)=\emptyset .
$$

Next, repeat the entire above argument, starting with the fact that the boundaries of the image of $M_{i+1}$ are disjoint from the boundaries of $N_{j+1}$. Continue inductively until a homeomorphism $h$ of $S^{3}$ to itself, fixed on $X \cup\left(S^{3}-M_{1}\right) \cup\left(S^{3}-N_{1}\right)$, is obtained, so that $h\left(\partial\left(M_{i+m}\right)\right) \cap \partial\left(N_{j+\ell}\right)=\emptyset$ for each nonnegative $m$ and $\ell$ less than $n$.

\section{Proof of the main Result}

As a special case, we first consider two Bing-Whitehead defining sequences for the same Bing-Whitehead compactum with the same initial stage.

Lemma 5.1. Assume that $X$ is a Bing-Whitehead compactum with two defining Bing-Whitehead sequences $\left(M_{i}\right)$ and $\left(N_{j}\right)$ and with $M_{0}=N_{0}$. Then there is a homeomorphism of $M_{0}=N_{0}$ that is fixed on $\partial M_{0}=\partial N_{0}$ and on $X$ that takes $M_{i}$ onto $N_{i}$ for any specified finite number of stages. In particular, if $X=B W\left(n_{1}, n_{2}, \ldots\right)$ with respect to $\left(M_{i}\right)$ and if $X=B W\left(m_{1}, m_{2}, \ldots\right)$ with respect to $\left(N_{j}\right)$, then $m_{i}=n_{i}$ for all $i$.

Proof. Suppose that such a homeomorphism $h_{n}$ exists that matches the components up through $n$ stages. Let $T$ be a component of $N_{n}$. Let $M$ equal $h_{n}\left(M_{n+1}\right) \cap T$ and $N$ equal $N_{n+1} \cap T$. By Lemma 4.2 we may assume that the boundaries of $M$ and $N$ are disjoint.

It must be the case that $M$ and $N$ both have the same number of components. To see this, suppose $M$ has one component and $N$ has two. If $M$ lies in a component of $N$, then the geometric index of $M$ in $T$ would be 0 instead of 2 . If $M$ does not lie in a component of $N, N$ must lie in the interior of $M$ and by Theorem 3.5. $M$ would be parallel to $\partial T$ and its geometric index in $T$ would be 1 instead of 2 .

In case $M$ and $N$ both have one component, suppose that $M$ lies in $N$. Then $\partial N$ is parallel to $\partial T$ or $\partial M$. But the geometric index of $N$ in $T$ is 2 , so $\partial M$ and $\partial N$ are parallel and the boundaries can be matched up with a homeomorphism of $T$ taking $\partial M$ to $\partial N$ fixed on $X$ and $\partial T$. The same argument works if $N$ lies in $M$.

Suppose now that $M$ and $N$ both have two components. Then one component of $M$ contains or is contained in one component of $N$, and the other component of $M$ contains or is contained in the other component of $N$. Theorem 3.5 can be used to show that $\partial M$ and $\partial N$ are parallel and as before we can get a homeomorphism fixed on $X$ and $\partial T$ taking $M$ to $N$.

Repeating this argument in each component of $N_{n}$ gives the homeomorphism $h_{n+1}$.

Note that the above proof also establishes the following lemma.

Lemma 5.2. Assume that $X$ is a Bing-Whitehead compactum with two defining sequences $\left(M_{i}\right)$ and $\left(N_{j}\right)$. If some component $M$ of $M_{i}$ is the same as some component $N$ of $N_{j}$, then for all $k>0, \alpha_{i+k}=\beta_{j+k}$, where $\left(\alpha_{\ell}\right)$ is the $B W$ pattern for $\left(M_{i}\right)$ and $\left(\beta_{\ell}\right)$ is the $B W$ pattern for $\left(N_{j}\right)$.

We next show that even without the same starting point, there is a component of some stage of one of the defining sequences that matches up with a component of the other defining sequence. 
Lemma 5.3. Assume that $X$ is a Bing-Whitehead compactum with two defining Bing-Whitehead sequences $\left(M_{i}\right)$ and $\left(N_{j}\right)$. Let stages $M_{m}$ and $N_{n}$ be chosen so that they miss the compactum at infinity of the other stage. Suppose that $T$ is a component of $N_{n}$ in the interior of some component of $M_{m}$. Then there is a homeomorphism of $S^{3}$, fixed on $X$, taking $T$ homeomorphically onto a component of some stage of $M_{m+\ell}$ for some $\ell \geq 0$.

Proof. We choose a $k$ so that $M_{m+k} \subset \operatorname{Int} N_{n}$. By Theorem 4.1 we may assume that $\partial T$ misses $\partial M_{i}, m \leq i \leq m+k$. Since $T \subset M_{m}$ and $T$ does not lie in a component of $M_{m+k}$, we can find the largest subscript $r$ so that $T$ does lie in a component of $M_{r}$. Let $S_{0}$ be the component of $M_{r}$ that contains $T$ and let $S_{1}$ be $M_{r+1} \cap S_{0}$. So $S_{1}$ is either a Bing link or a Whitehead link in $S_{0}$. If $S_{1}$ is a Whitehead link, then $S_{1} \subset \operatorname{Int} T$ and, by Theorem 3.4 $\partial T$ is boundary parallel to either $\partial S_{0}$ or $\partial S_{1}$. In this case we may now assume by a homeomorphism fixing $X$ that $T$ equals $S_{0}$ or $S_{1}$. In case $S_{1}$ is a Bing link, then at least one and possibly both components of $S_{1}$ lie in $\operatorname{Int} T$. If both lie in $\operatorname{Int} T$, then $\partial T$ and $\partial S_{0}$ are parallel by Theorem 3.5. If one component $S_{1}^{\prime} \subset \operatorname{Int} T$ and the other component misses $T$, then $\partial T$ and $\partial S_{1}^{\prime}$ are parallel by Theorem 3.6. In either case, we may assume by a homeomorphism fixing $X$ that $T$ is either $S_{0}$ or $S_{1}^{\prime}$.

The previous lemmas can now be used to provide a proof of the main theorem.

Proof of Theorem 2.5. Let $X_{1}$ be a Bing-Whitehead Cantor set associated with a defining sequence $\left(M_{i}\right)$ and let $X_{2}$ be a Bing-Whitehead Cantor set associated with a defining sequence $\left(N_{j}\right)$. Assume that $X_{1}$ and $X_{2}$ are equivalently embedded. Then there is a homeomorphism of $S^{3}$ taking $X_{1}$ to $X_{2}$, so without loss of generality, we may assume $X=X_{1}=X_{2}$ and that $X$ has two Bing-Whitehead defining sequences $\left(M_{i}\right)$ and $\left(N_{j}\right)$. Let $\left(\alpha_{1}, \alpha_{2}, \alpha_{3}, \ldots\right)$ be the BW pattern of $X$ with respect to $\left(M_{i}\right)$ and let $\left(\beta_{1}, \beta_{2}, \beta_{3}, \ldots\right)$ be the BW pattern of $X$ with respect to $\left(N_{j}\right)$. Choose stages $M_{m}$ of $\left(M_{i}\right)$ and $N_{n}$ of $\left(N_{j}\right)$ so that

- $M_{m}$ is contained in $N_{1}$ and $N_{n}$ is contained in $M_{1}$.

- Both $M_{m}$ and $N_{n}$ have $2^{r}$ components, and both $M_{m+1}$ and $N_{n+1}$ are obtained by placing Bing constructions in each component of the previous stage.

Apply Lemma 4.2 to adjust $M_{m}$ and $N_{n}$ so that their boundaries do not intersect. If all the components of $M_{m}$ are contained in components of $N_{n}$, then the components must match up in a 1-1 fashion, and the proof of Lemma 5.3, together with the fact that the next stage is a Bing construction, shows that there is a homeomorphism matching up these components. Then by Lemma 5.2 $\beta_{n+k}=\alpha_{m+k}$ for all $k \geq 0$, establishing the needed result. A similar argument gives this conclusion if all the components of $N_{n}$ are contained in components of $M_{m}$.

If some component of $M_{m}$ contains more than one component of $N_{n}$, then some component of $N_{n}$ also contains more than one component of $M_{m}$. Let $T_{1}$ be a component of $N_{n}$ contained in some component of $M_{m}$. By Lemma 5.3 $T_{1}$ can be matched homeomorphically with a component of some $M_{m+p}$ and so by Lemma 5.2. $\beta_{n+k}=\alpha_{m+p+k}$ for all $k \geq 0$. Let $T_{2}$ be a component of $M_{m}$ contained in some component of $N_{n}$. By Lemma [5.3, $T_{2}$ can be matched homeomorphically with a component of some $N_{n+q}$ and so by Lemma 5.2. $\alpha_{m+k}=\beta_{n+q+k}$ for all $k \geq 0$. Thus

$$
\alpha_{m+k}=\beta_{n+q+k}=\alpha_{m+p+q+k}=\alpha_{(m+k)+(p+q)} .
$$


If $p>0$ or $q>0$, this implies that the BW pattern for $X$ with respect to $\left(M_{i}\right)$ is repeating, contradicting the fact that $\sum_{i} n_{i} 2^{-i}$ diverges where $X=B W\left(n_{1}, n_{2}, \ldots\right)$ with respect to $\left(M_{i}\right)$. Thus $p=q=0$ and $\beta_{n+k}=\alpha_{m+k}$ for all $k \geq 0$, establishing the needed result.

\section{Questions}

(1) Is it possible to generalize the main theorem (Theorem 2.5) to apply to the construction of DeGryse and Osborne in dimensions greater than three?

(2) Is it possible to distinguish Bing-Whitehead compacta that vary the placement of Bing and Whitehead constructions at each stage, rather than using all Bing or all Whitehead constructions at each stage?

(3) Is it possible to use the techniques of the main theorem to construct rigid Cantor sets of genus one in $S^{3}$ with simply connected complements? See [GRŽ06] for a discussion of rigid Cantor Sets.

\section{ACKNOWLEDGMENTS}

The authors would like to thank the referee for helpful suggestions. The authors were supported in part by the Slovenian Research Agency grants No.P1-509-0101, J1-9643-0101 and BI-US/08-09-003. The first author was supported in part by the National Science Foundation grant DMS0453304. The first and third authors were supported in part by the National Science Foundation grant DMS0707489. The second and the fourth authors were supported in part by the Slovenian Research Agency grants P1-0292-0101 and J1-9643-0101.

\section{REFERENCES}

[Ant20] M. L. Antoine, Sur la possibilité d'étendre l'homeomorphie de deux figures à leur voisinages, C.R. Acad. Sci. Paris 171 (1920), 661-663.

[AS89] Fredric D. Ancel and Michael P. Starbird, The shrinkability of Bing-Whitehead decompositions, Topology 28 (1989), no. 3, 291-304. MR1014463 (90g:57014)

[BC87] M. Bestvina and D. Cooper, A wild Cantor set as the limit set of a conformal group action on $S^{3}$, Proc. Amer. Math. Soc. 99 (1987), no. 4, 623-626. MR877028(88b:57015)

[Bla51] William A. Blankinship, Generalization of a construction of Antoine, Ann. of Math. (2) 53 (1951), 276-297. MR $12: 730 \mathrm{c}$

[Dav86] Robert J. Daverman, Decompositions of manifolds, Pure and Applied Mathematics, vol. 124, Academic Press Inc., Orlando, FL, 1986. MR872468 (88a:57001)

[DO74] D. G. DeGryse and R. P. Osborne, A wild Cantor set in $E^{n}$ with simply connected complement, Fund. Math. 86 (1974), 9-27. MR0375323 (51:11518)

[GR07] Dennis J. Garity and Dušan Repovš, Cantor set problems, Open problems in topology. II. (Elliott Pearl, ed.), Elsevier B. V., Amsterdam, 2007, pp. 676-678. MR 2367385 (2008j:54001)

[GRŽ05] D. Garity, D. Repovš, and M. Željko, Uncountably many Inequivalent Lipschitz Homogeneous Cantor sets in $R^{3}$, Pacific J. Math. 222 (2005), no. 2, 287-299. MR2225073 (2006m:54056)

[GRŽo6] , Rigid Cantor sets in $R^{3}$ with Simply Connected Complement, Proc. Amer. Math. Soc. 134 (2006), no. 8, 2447-2456. MR2213719 (2007a:54020)

[Kir58] A. Kirkor, Wild 0-dimensional sets and the fundamental group, Fund. Math. 45 (1958), 228-236. MR0102783 (21:1569)

[Mye88] Robert Myers, Contractible open 3-manifolds which are not covering spaces, Topology 27 (1988), no. 1, 27-35. MR 935526 (89c:57012)

[Mye99a] _ Contractible open 3-manifolds which non-trivially cover only non-compact 3manifolds, Topology 38 (1999), no. 1, 85-94. MR1644087 (99g:57022) 
[Mye99b] _ Contractible open 3-manifolds with free covering translation groups, Topology Appl. 96 (1999), no. 2, 97-108. MR1702304(2001a:57031)

[Mye00a] _ Compactifying sufficiently regular covering spaces of compact 3-manifolds, Proc. Amer. Math. Soc. 128 (2000), no. 5, 1507-1513. MR1637416 (2000j:57050)

[Mye00b] _ On covering translations and homeotopy groups of contractible open $n$ manifolds, Proc. Amer. Math. Soc. 128 (2000), no. 5, 1563-1566. MR.1641077 (2001a:57005)

[Mye00c] Uncountably many arcs in $S^{3}$ whose complements have non-isomorphic, indecomposable fundamental groups, J. Knot Theory Ramifications 9 (2000), no. 4, 505-521. MR:1758869 (2001m:57014)

[Rol76] D. Rolfsen, Knots and links, Mathematics Lecture Series, No. 7., Publish or Perish, Inc., Berkeley, Calif., 1976. MR0515288 (58:24236)

[RS72] C. P. Rourke and B. J. Sanderson, Introduction to piecewise-linear topology, SpringerVerlag, New York, 1972, Ergebnisse der Mathematik und ihrer Grenzgebiete, Band 69. MR0350744 (50:3236)

[Sch53] H. Schubert, Knoten und vollringe, Acta. Math. 90 (1953), 131-186. MR0072482 $(17,291 \mathrm{~d})$

[She68] R. B. Sher, Concerning wild Cantor sets in $E^{3}$, Proc. Amer. Math. Soc. 19 (1968), 1195-1200. MR 38:2755

[Shi74] A. C. Shilepsky, A rigid Cantor set in $E^{3}$, Bull. Acad. Polon. Sci. Sér. Sci. Math. 22 (1974), 223-224. MR0345110 (49:9849)

[Sko86] Richard Skora, Cantor sets in $S^{3}$ with simply connected complements, Topology Appl. 24 (1986), no. 1-3, 181-188, Special volume in honor of R. H. Bing (1914-1986). MR872489 (87m:57009)

[Wri89] David G. Wright, Bing-Whitehead Cantor sets, Fund. Math. 132 (1989), no. 2, 105-116. MR1002625 (90d:57020)

[Wri92] , Contractible open manifolds which are not covering spaces, Topology 31 (1992), no. 2, 281-291. MR 93f:57004

[Žel00] Matjaž Željko, On Embeddings of Cantor Sets into Euclidean spaces, Ph.D. thesis, University of Ljubljana, Ljubljana, Slovenia, 2000.

[Žel01] _ On defining sequences for Cantor sets, Topology Appl. 113 (2001), no. 1-3, 321-325, Geometric topology: Dubrovnik 1998. MR.1821859 (2002d:57013)

[Žel05] _ Genus of a Cantor set, Rocky Mountain J. Math. 35 (2005), no. 1, 349-366. MR2117612 (2006e:57022)

Department of Mathematics, Oregon State University, Corvallis, Oregon 97331

E-mail address: garity@math.oregonstate.edu

Faculty of Mathematics and Physics, and Faculty of Education, University of LjublJana, P.O. Box 2964, Luubluana, Slovenia 1001

E-mail address: dusan.repovs@guest.arnes.si

Department of Mathematics, Brigham Young University, Provo, Utah 84602

E-mail address: wright@math.byu.edu

Institute of Mathematics, Physics and Mechanics, Faculty of Mathematics and Physics, University of Ljubluana, P.O.Box 2964, Luubluana, Slovenia

E-mail address: matjaz.zeljko@fmf.uni-lj.si 